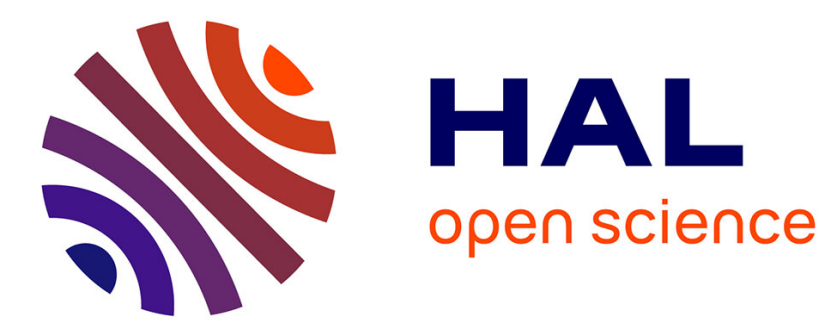

\title{
Entry game under opportunistic access in cognitive radio networks: a priority queue model
}

\author{
Luis Guijarro, Vicent Pla, Bruno Tuffin
}

\section{To cite this version:}

Luis Guijarro, Vicent Pla, Bruno Tuffin. Entry game under opportunistic access in cognitive radio networks: a priority queue model. IFIP Wireless days, Nov 2013, Valencia, Spain. hal-00908585

\section{HAL Id: hal-00908585}

\section{https://hal.inria.fr/hal-00908585}

Submitted on 25 Nov 2013

HAL is a multi-disciplinary open access archive for the deposit and dissemination of scientific research documents, whether they are published or not. The documents may come from teaching and research institutions in France or abroad, or from public or private research centers.
L'archive ouverte pluridisciplinaire HAL, est destinée au dépôt et à la diffusion de documents scientifiques de niveau recherche, publiés ou non, émanant des établissements d'enseignement et de recherche français ou étrangers, des laboratoires publics ou privés. 


\title{
Entry game under opportunistic access in cognitive radio networks: a priority queue model
}

\author{
Luis Guijarro*, Vicent Pla* and Bruno Tuffin ${ }^{\dagger}$ \\ *Universitat Politècnica de València, Spain \\ †nria, France
}

\begin{abstract}
A cognitive radio network where an incumbent primary operator and an entrant secondary operator compete for users is modeled using queueing and game theory. The economic viability of supporting the secondary operator service using an opportunistic access to the spectrum owned by the primary operator is assessed. Against the benchmark of the primary operator operating as a monopolist, we show that the entry of the secondary operator is desirable from an efficiency perspective, since the carried traffic increases. Additionally, for a range of parameter values, a lump sum payment can be designed so that the incumbent operator has an incentive to let the secondary operator enter. Additionally, the opportunistic access setting has been compared against a leasing-based alternative, and we have concluded that the former outperforms the latter in terms of efficiency and incentive.
\end{abstract}

\section{INTRODUCTION}

Cognitive radio technologies for spectrum sharing have received an enormous interest from the research community for the last decade, and more recently from regulators and mobile operators [1]. Whichever technical solution will be finally deployed, there is an agreement among regulators and operators that spectrum sharing opportunities will only emerge if both quality of service guarantees and market-based incentives are agreed between sharing partners [2]. This paper focuses on the latter, that is, on the economical transactions that will provide incentives to mobile operators to engage in cognitive radio-based spectrum sharing schemes.

There are some contributions on the modeling of the economical interactions between primary and secondary operators in cognitive radio networks. Reference [3] analyzes spectrum leasing in cognitive radio networks and derives primary profitmaximizing pricing schemes for the leasing transaction; there is no explicit modeling of the secondary operator. Reference [4] (respectively, Reference [5]) analyzes the spectrum leasing transaction between one primary operator and many secondary operators (respectively, many primary operators and one secondary operator), under a user behavior modeled by a demand function. However, primary and secondary operators are providing services in disjoint markets, so that no interaction in the service provision is modeled. We are concerned with the interaction between primary and secondary operators at both the operational and the economincal level. Actually, we have previously worked in such sort of models in [6] and [7], where spectrum leasing was agreed between a primary and a secondary operator, and then service competition took place. Still, once the leasing transaction was completed, there would be no further cross effect on the quality of the service provided by the operators.
Our focus in this paper is to proceed one step further and model both the economical interaction, by means of price competition, and the operational interaction-as we describe in next section, we use priority queueing for the latter. There are many works on the provision of differentiated service by means of priority queueing, motivated by seminal papers such as [8]. The role of pricing in differentiated servicing is analyzed in papers such as [9] and [10]. We are indebted specifically to [11], [12] and [13]. The first one analyzes pricing in a communications network; it investigates priority queueing as a way to differentiate between users with heterogeneous service requirements. The other ones extend the model to analyze the effects of using processor-sharing discriplines. However, these works only model one network operator, that is, the competition pressure is absent. Instead, we explicitly model competing operators which extract revenues flow from the service that each priority class receives. This model requires a different analysis compared with the above papers.

The paper is structured as follows. Next section describes the queueing model and the payment relationships, whereas Section III describes the specific game that is used to model the interaction. Section IV analyzes the baseline case where the primary operator is a monopolist, and Section $\mathrm{V}$ analyzes the dupoly case, computes numerical values, and compares the duopoly and the monopoly cases. Section VI analyzes an alternative setting with spectrum leasing and no opportunistic access, and finally Section VII draws the conclusions.

\section{General MODEL}

We model a duopoly where mobile communication users pay for the packet-delivery service offered by two competing operators. One of the operators holds a spectrum license, and the other one does not. The latter coordinates subscribers access to the spectrum licensed to the former in an opportunistic manner using cognitive radio technologies. Hence, we denote the former as either incumbent or primary operator, and the latter, as entrant or secondary operator.

We assume that the spectrum access implemented and coordinated by the secondary operator does not cause a significant impact in the quality of service received by primary operator's subscribers. Therefore, we model this service delivery using a single-server Priority Queue (Fig. 1), where the users that pay for primary operator's service are priority users, and those ones that pay for secondary operator's service are ordinary users. In such a queue, each class (priority and ordinary) has a separate (logical) queue, and when the server becomes free, the user from the head of the priority-class queue, if non-empty, enters the server and it is served. Still, the primary users may suffer 


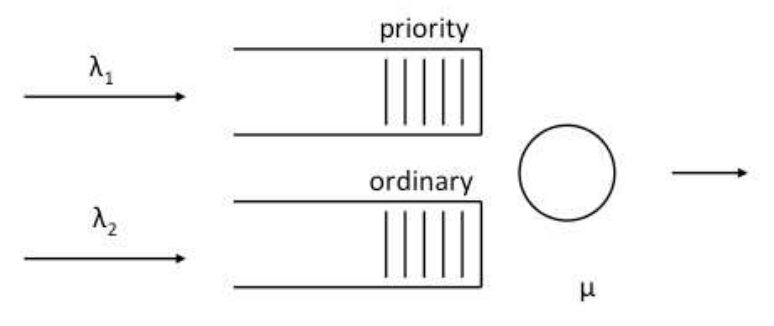

Figure 1. Queue model for the opportunistic access

an impaired performance due to failures in the opportunistic access coordinated by the secondary operator, e.g. spectrum sensing limitations and delays. We take this impairment into account through a non-preemptive variation of the priorityqueue discipline, where the service of the user being served is always completed, even if priority users may arrive meanwhile. Finally, we use a First-Come-Fist-Served discipline, although a Processor Sharing discipline within each priority class could be easily incorporated in the model.

In our model, the users are the packets to be transmitted; hence, priority (resp. ordinary) packets are those packets that pay to the primary (resp. secondary) operator and are queued in the priority (resp. ordinary) queue. We assume that priority packets (resp. ordinary packets) arrive to the system according to a Poisson process with rate $\lambda_{1}$ (resp. $\lambda_{2}$ ), and the transmission time of all packets is exponentially distributed with mean $\bar{x}=1 / \mu$. The packets wait for service in a queue with infinite length. Using Kendall notation, our system is an $\mathrm{M} / \mathrm{M} / 1$ queue. Packets are not only homogeneous in their service time requirements, but also in their preferences on the timeliness of the service. Specifically, we model the quality a packet gets from the time it takes to complete transmission if it has paid to Operator $i$ ( $i=1$ for the primary operator, and $i=2$ for the secondary operator) as $Q_{i}=c \cdot T_{i}^{-\alpha}$, where $T_{i}$ is the mean service time and $0<\alpha<1$.

As regards the operators, each one charges a price for transmitting a packet, that is, the primary operator charges a price $p_{1}$ to each priority packet, and the secondary operator charges a price $p_{2}$ to each ordinary packet. We assume that no cost is incurred by the operators, so that Operator $i$ 's profit $\Pi_{i}$ is given by the revenue flow $\lambda_{i} p_{i}$.

The rate at which priority packets (resp. ordinary packets) arrive at the system and pay for service will then be guided by the price $p_{1}$ charged by the primary operator (resp. secondary operator) and the quality $Q_{1}$ (resp. $\left.Q_{2}\right)$. Flow $i(i=1$ for the priority packets, and $i=2$ for the ordinary packets) will adjust its rate $\lambda_{i}$ so that the utility

$$
u_{i} \triangleq Q_{i}-p_{i}
$$

is greater than or equal to zero-we assume that packets get zero utility from not being transmitted. We call the adjustment of $\lambda_{i}$ the flow $i$ 's subscription decision. The pricing decisioni.e, the selection of $p_{i}$-is taken by each operator independently from each other so as to maximizing its profit $\Pi_{i}$.

\section{GAME MODEL}

From the model description above we can observe the following strategic interactions:

- Flow $i$ 's subscription decision is influenced by Operator $i$ 's pricing decision.

- Operator $i$ 's profit is influenced by Flow $i$ 's subscription decision.

- $\quad$ Flow $i$ 's subscription decision is also influenced by Flow $j$ 's decision $(j \neq i)$, through the $Q_{i}$ factor.

- Operator $i$ 's profit is influenced by Operator $j$ 's pricing decision, indirectly through Flow $j$ 's subscription decision.

We conclude then that the above strategic scenario is amenable to game theory. Specifically, we will use a two-stage sequential game of the multi-leader-follower kind, where (1) in the first stage, each operator chooses its pricing strategy $p_{i}$ in order to maximize its profit $\Pi_{i}$; and (2) in the second stage, each packet flow takes its own subscription decision, trying to maximize the utility it gets from either subscribing or not.

In the second stage, each flow observes the price posted by its serving operator and then makes its decision. Assuming that the number of packets arriving during one time period is high enough, the individual subscription decision of one packet does not affect the utility that the rest of the packets from either flow get. Under this setting, the equilibrium will be reached as postulated by Wardrop ${ }^{1}$. Applying this notion of equilibrium, known as Wardrop equilibrium, to the flow subscription problem, we postulate that $\lambda_{i}^{*}$ (or equivalently, $\rho_{i}^{*}=\lambda_{i}^{*} \bar{x}$ ) will be such that either $\rho_{i}>0$ and $u_{i}=0$; or $\rho_{i}=0$ and $u_{i}<0$. Therefore, four cases result::

Case $1 \quad u_{1}=0$ and $u_{2}=0$, so that

$$
Q_{i}-p_{i}=0 \quad i=1,2
$$

Case $2 u_{1}=0$ and $u_{2}<0$, in particular, $\rho_{2}=0$

Case $3 u_{1}<0$ and $u_{2}=0$, in particular, $\rho_{1}=0$

Case $4 \quad u_{1}<0$ and $u_{2}<0$, in particular, $\rho_{1}=\rho_{2}=0$

In the first stage, the two operators choose their respective prices in a simultaneous and independent way. Each operator is not only aware of the flow subscription decision in the second stage, but also of the rational behavior of the competing operator, who is choosing its strategy at the same stage.

Under this setting, the equilibrium concept is the Subgame Perfect Nash Equilibrium (SPNE) [15], where, given that the flows will optimally decide as postulated above, the pricing strategy chosen by each operator will be such that

$$
\begin{aligned}
& p_{1}^{*}=\underset{p_{1}}{\arg \max } \Pi_{1}\left(p_{1}, p_{2}^{*}\right) \\
& p_{2}^{*}=\underset{p_{2}}{\arg \max } \Pi_{2}\left(p_{1}^{*}, p_{2}\right) .
\end{aligned}
$$

${ }^{1}$ Within the context of trasport networks, and assuming that users choose routes trying to minimize travel costs, Wardrop's first principle states: The journey times in all routes actually used are equal and less than those which would be experienced by a single vehicle on any unused route [14]. 


\section{MONOPOLY}

We begin by the case where there is only one operator providing service, namely, the primary operator, so that no priority is implemented and the operator behaves as a monopolist. This case will be used as a benchmark for the analysis of the dupoly scenario.

The queue model is thus an $\mathrm{M} / \mathrm{M} / 1$ queue with a packet traffic of $\rho=\lambda \bar{x}$. The expression for the mean service time is then

$$
T=\frac{\rho \bar{x}}{1-\rho \theta}+\bar{x}=\frac{\bar{x}}{1-\rho},
$$

so that the quality is

$$
Q(\rho)=c\left(\frac{\bar{x}}{1-\rho}\right)^{-\alpha}
$$

and the utility is $u=Q-p$.

We postulate that, given a price $p$ charged by the monopolist operator, the (Wardrop) equilibrium results in the following two alternatives:

Case 1 The amount of packets that pay for service increases until the utility they get drops to zero and therefore packets are indifferent between either being transmitted or not. Actually, taking the first derivative of $u$ with respect to $\rho$, we check that $\frac{\partial u}{\partial \rho}<0$. The value $\rho$ which results in $u=0$ will be such that

$$
Q(\rho)-p=0 .
$$

Case 2 The price is so high that no packet pays for service, that is $u<0$ and $\rho=0$. This happens when $Q(0)-p<0$, or $c \bar{x}^{-\alpha}<p$.

The monopolist operator has a revenue of

$$
\Pi=\lambda p=\frac{\rho}{\bar{x}} p .
$$

Given that the traffic reaches the Wardrop equilibrium, the monopolist will choose $p$ so as to maximize its revenue. For this, the first derivative of (8) should be equal to zero:

$$
\frac{\partial \Pi}{\partial p}=\frac{1}{\bar{x}}\left(\rho+p \frac{\partial \rho}{\partial p}\right)=0
$$

Let us first assume that the user equilibrium is as stated in Case 1. Taking the first derivative of (7) with respecto to $p$

$$
\frac{\partial Q}{\partial \rho} \frac{\partial \rho}{\partial p}-1=0
$$

and using (9), we end up with an equation on $\rho$,

$$
Q+\rho \frac{\partial Q}{\partial \rho}=0
$$

Substituting (6), we can solve (11) for the equilibrium traffic

$$
\rho_{m}=\frac{1}{1+\alpha}
$$

which is lower that 1 .
Using (10) and (9), we can compute the profit-maximizing price as

$$
p_{m}=-\rho_{m} \frac{\partial Q}{\partial \rho}=c\left(\frac{\alpha}{\bar{x}(1+\alpha)}\right)^{\alpha}=c \bar{x}^{-\alpha}\left(\frac{\alpha}{1+\alpha}\right)^{\alpha} .
$$

Note that $p_{m}<c \bar{x}^{-\alpha}$, consistent with assuming Case 1. And the monopoly profit as

$$
\Pi_{m}=\frac{\rho_{m}}{\bar{x}} p_{m}=c \bar{x}^{-(1+\alpha)} \frac{\alpha^{\alpha}}{(1+\alpha)^{1+\alpha}} .
$$

\section{DUOPOLY}

In this section we analyze the case where the primary and secondary operator are both operating in the market. We proceed to derive the equilibrium values for the priority and ordinary traffic, and the operators' prices and profits.

Let us define, for each flow $i=1,2$, the mean service time $T_{i} \triangleq W_{i}+T_{i}^{\prime}$, where $W_{i}$ is the mean waiting time until transmission begins, and $T_{i}^{\prime}$, the mean transmission time. And the quantities $\rho_{i} \triangleq \lambda_{i} \bar{x}$. Let us compute the above quantities for the $\mathrm{M} / \mathrm{M} / 1$ non-preemptive priority queue [16]. The residual service time for all packets is $R=\left(\rho_{1}+\rho_{2}\right) \bar{x}$. The mean waiting time for each flow is given by

$$
\begin{aligned}
& W_{1}=\frac{R}{1-\rho_{1}} \\
& W_{2}=\frac{R}{\left(1-\rho_{1}\right)\left(1-\left(\rho_{1}+\rho_{2}\right)\right)}
\end{aligned}
$$

and $T_{i}^{\prime}=\bar{x}$, since preemption is not allowed. The expressions for the mean service times are then

$$
\begin{aligned}
& T_{1}=\frac{1+\rho_{2}}{1-\rho_{1}} \bar{x} \\
& T_{2}=\frac{1-\rho_{1}\left(1-\left(\rho_{1}+\rho_{2}\right)\right)}{\left(1-\rho_{1}\right)\left(1-\left(\rho_{1}+\rho_{2}\right)\right)} \bar{x} .
\end{aligned}
$$

And the expressions for the quality are

$$
\begin{aligned}
Q_{1}\left(\rho_{1}, \rho_{2}\right) & =c \frac{\left(1-\rho_{1}\right)^{\alpha}}{\left(1+\rho_{2}\right)^{\alpha}} \bar{x}^{-\alpha} \\
Q_{2}\left(\rho_{1}, \rho_{2}\right) & =c \frac{\left(1-\rho_{1}\right)^{\alpha}\left(1-\left(\rho_{1}+\rho_{2}\right)\right)^{\alpha}}{\left(1-\rho_{1}\left(1-\left(\rho_{1}+\rho_{2}\right)\right)\right)^{\alpha}} \bar{x}^{-\alpha} .
\end{aligned}
$$

\section{A. User equilibrium}

We obtain the equilibrium values for $\rho_{1}$ and $\rho_{2}$, for given values of $p_{1}$ and $p_{2}$ according to the Wardrop equilibria stated in Section III.

1) Case 1: The conditions $u_{1}=0$ and $u_{2}=0$ give equilibrium values $\rho_{1}$ and $\rho_{2}$ such that $Q_{1}\left(\rho_{1}, \rho_{2}\right)-p_{1}=0$ and $Q_{2}\left(\rho_{1}, \rho_{2}\right)-p_{2}=0$.

2) Case 2: Since $\rho_{2}=0,(19)$ and (20) become

$$
\begin{aligned}
& Q_{1}\left(\rho_{1}, 0\right)=c\left(1-\rho_{1}\right)^{\alpha} \bar{x}^{-\alpha} \\
& Q_{2}\left(\rho_{1}, 0\right)=c \frac{\left(1-\rho_{1}\right)^{2 \alpha}}{\left(1-\rho_{1}\left(1-\rho_{1}\right)\right)^{\alpha}} \bar{x}^{-\alpha} .
\end{aligned}
$$

Solving $u_{1}=Q_{1}\left(\rho_{1}, 0\right)-p_{1}=0$ for $\rho_{1}$, we obtain

$$
\rho_{1,0}=1-\bar{x}\left(\frac{p_{1}}{c}\right)^{1 / \alpha} \text {. }
$$


On the other hand, the condition $u_{2}<0$ is held for $p_{2}>\hat{p}_{2}=Q_{2}\left(\rho_{1,0}, 0\right)=c \frac{\bar{x}^{2 \alpha}\left(\frac{p_{1}}{c}\right)^{2}}{\left(1-\left(1-\bar{x}\left(\frac{p_{1}}{c}\right)^{1 / \alpha}\right) \bar{x}\left(\frac{p_{1}}{c}\right)^{1 / \alpha}\right)^{\alpha}} \bar{x}^{-\alpha}$.

Note that $\rho_{2}=0$ means that Operator 2's profit is zero.

3) Case 3: Since $\rho_{1}=0,(19)$ and (20) become

$$
\begin{aligned}
& Q_{1}\left(0, \rho_{2}\right)=c\left(1+\rho_{2}\right)^{-\alpha} \bar{x}^{-\alpha} \\
& Q_{2}\left(0, \rho_{2}\right)=c\left(1-\rho_{2}\right)^{\alpha} \bar{x}^{-\alpha} .
\end{aligned}
$$

Solving $u_{2}=Q_{2}\left(0, \rho_{2}\right)-p_{2}=0$ for $\rho_{2}$, we obtain

$$
\rho_{2,0}=1-\bar{x}\left(\frac{p_{2}}{c}\right)^{1 / \alpha} \text {. }
$$

On the other hand, the condition $u_{1}<0$ is held for

$$
p_{1}>\hat{p}_{1}=Q_{1}\left(0, \rho_{2,0}\right)=c\left(2-\bar{x}\left(\frac{p_{2}}{c}\right)^{1 / \alpha}\right)^{-\alpha} \bar{x}^{-\alpha} .
$$

Note that $\rho_{1}=0$ means that Operator 1's profit is zero.

4) Case 4: Finally, assuming $\rho_{1}=\rho_{2}=0$, (19) and (20) become

$$
\begin{aligned}
& Q_{1}(0,0)=c \bar{x}^{-\alpha} \\
& Q_{2}(0,0)=c \bar{x}^{-\alpha}
\end{aligned}
$$

The condition $u_{1}<0$ is held for $p_{1}>Q_{1}(0,0)=c \bar{x}^{-\alpha}$. The same applies to $p_{2}$, that is, $p_{2}>c \bar{x}^{-\alpha}$. Note that $\rho_{1}=$ $\rho_{2}=0$ means that both operator's profits are zero.

As a corollary, price values for Case 1 are defined by the opposite of (28) and (24), that is,

$$
p_{1} \leq \hat{p}_{1} \quad p_{2} \leq \hat{p}_{2} .
$$

\section{B. Subgame Perfect Nash Equilibrium}

We proceed to compute the equilibrium values for $p_{1}$ and $p_{2}$, assuming that the values for $\rho_{1}$ and $\rho_{2}$ (for given values $p_{1}$ and $p_{2}$ ) are the ones from the previous subsection.

Let us first assume that Case 1 holds. We use (2) and we compute its first derivative, so that for each packet flow $i$

$$
\frac{\partial Q_{i}}{\partial \rho_{i}} \frac{\partial \rho_{i}}{\partial p_{i}}-1=0
$$

On the other hand, from (4), the first-order condition for Operator $i$ results in

$$
\frac{\partial \Pi_{i}}{\partial p_{i}}=\frac{1}{\bar{x}}\left(\rho_{i}+p_{i} \frac{\partial \rho_{i}}{\partial p_{i}}\right)=0 .
$$

Proceeding in a similar way to Section IV, we can obtain the equilibrium traffic $\rho_{i}$ for packet flow $i$ from

$$
Q_{i}+\rho_{i} \frac{\partial Q_{i}}{\partial \rho_{i}}=0
$$

and compute the equilibrium price $p_{i}$ and profit $\Pi_{i}$ for Operator $i$ as

$$
\begin{aligned}
p_{i} & =-\rho_{i} \frac{\partial Q_{i}}{\partial \rho_{i}} \\
\Pi_{i} & =\frac{\rho_{i}}{\bar{x}} p_{i} .
\end{aligned}
$$

The first partial derivatives of (19) and (20) are

$$
\begin{aligned}
& \frac{\partial Q_{1}}{\partial \rho_{1}}=c \frac{\bar{x}^{-\alpha}}{\left(1+\rho_{2}\right)^{\alpha}} \alpha\left(1-\rho_{1}\right)^{\alpha-1}(-1) \\
& \frac{\partial Q_{2}}{\partial \rho_{2}}=-c\left(1-\rho_{1}\right)^{\alpha} \bar{x}^{-\alpha} \alpha \frac{\left(1-\left(\rho_{1}+\rho_{2}\right)\right)^{\alpha-1}}{\left(1-\rho_{1}\left(1-\left(\rho_{1}+\rho_{2}\right)\right)\right)^{\alpha+1}} .
\end{aligned}
$$

Solving (34) for operator 1 gives

$$
\rho_{1}=\frac{1}{1+\alpha}
$$

while the solution for operator 2 is

$$
\rho_{2}=\frac{1+\alpha-2 \rho_{1}+2 \rho_{1}^{2}-\sqrt{1+2 \alpha-4 \alpha \rho_{1}+4 \alpha \rho_{1}^{2}+\alpha^{2}}}{-2 \rho_{1}} .
$$

Finally, values for prices and profits can be computed respectively from (35) and (36), using (37) and (38).

Note that $\rho_{1}$ and $\rho_{2}$ are positive, and we will check numerically in next section that first, equilibrium prices comply with (31) and, second, profits are also positive. This rules out that the SPNE lies in either Case 4 (since either operator will raise its profit by reducing its price below $c \bar{x}^{-\alpha}$ ), Case 2 (since operator 2 will raise its profit by reducing its price below $\hat{p}_{2}$ ) or Case 3 (since operator 1 will raise its profit by reducing its price below $\left.\hat{p}_{1}\right)$.

\section{Results and discussion}

In this section, numerical values are computed for the equilibrium traffic, prices, and profits for the primary and secondary operators. The values are compared against the monopoly scenario.

We set parameters $\bar{x}=1$, and $c=1$, and we vary $\alpha \in[0.1,0.9]$. Fig. 2 shows the equilibrium traffic for the monopolist $\left(\rho_{m}\right)$, the primary $\left(\rho_{1}\right)$ and the secondary $\left(\rho_{2}\right)$ operators. It also shows $\rho_{1}+\rho_{2}$. Fig. 3 shows the equilibrium prices and Fig. 4 shows the equilibrium profits.

We can see that:

- The carried traffic by the primary operator is the same that would have carried if operating as a monopolist, i.e. $\rho_{1}=\rho_{m}$. Additionally, the system is able to accommodate ordinary packets (up to $\rho_{2}$ ) paying for service to the entrant (secondary) operator. This was analytically derived in (39) and (40).

- The primary operator suffers a decrease in its profits when a secondary operator enters the market.

- As $\alpha$ increases, the monopolist is able to carry less traffic, and therefore its profit decreases. This may be explained by the fact that, as $\alpha$ increases, a given mean service time value $T$ translates into a lower utility. 


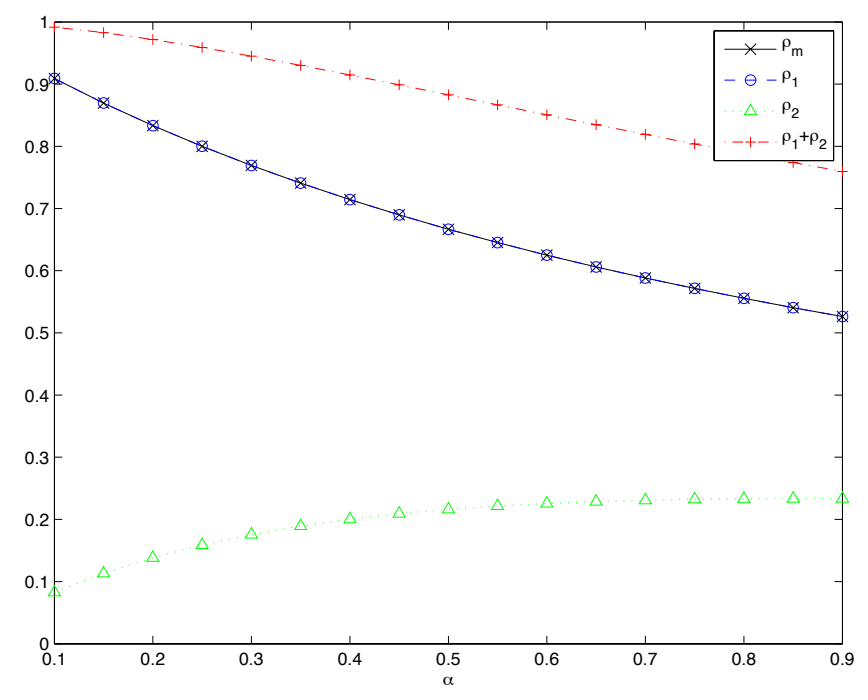

Figure 2. Equilibrium traffic values

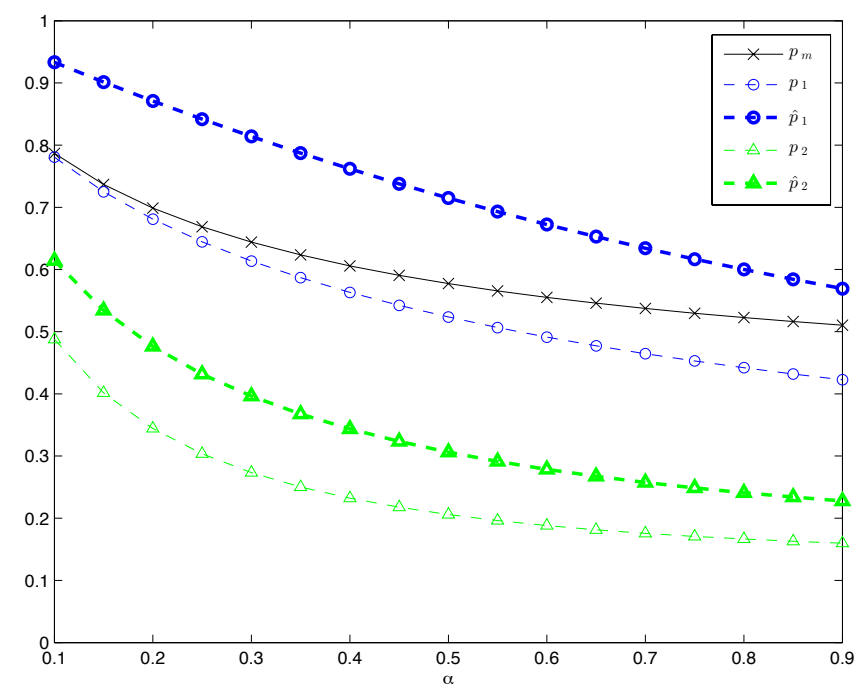

Figure 3. Equilibrium prices

- The effect of $\alpha$ on the primary operator traffic, price and profits is roughly the same as the effect on the monopolist.

- As $\alpha$ increases, the carried traffic by the secondary operator increases, but its profit increases only up to $\alpha \approx 0.25$.

- As $\alpha$ increases, the amount of total traffic that the duopoly can accommodate decreases, which is a similar effect as in the monopoly.

Fig. 3 also shows the threshold price $\hat{p}_{1}$ (resp. $\hat{p}_{2}$ ) which sets the maximum price for which Operator 1 (resp. Operator 2 ) would get positive profits. The graph shows that equilibrium prices $p_{1}$ and $p_{2}$ keep under these threshold values, confirming the assumption in Section V-B that SPNE is in the region defined by Case 1 .

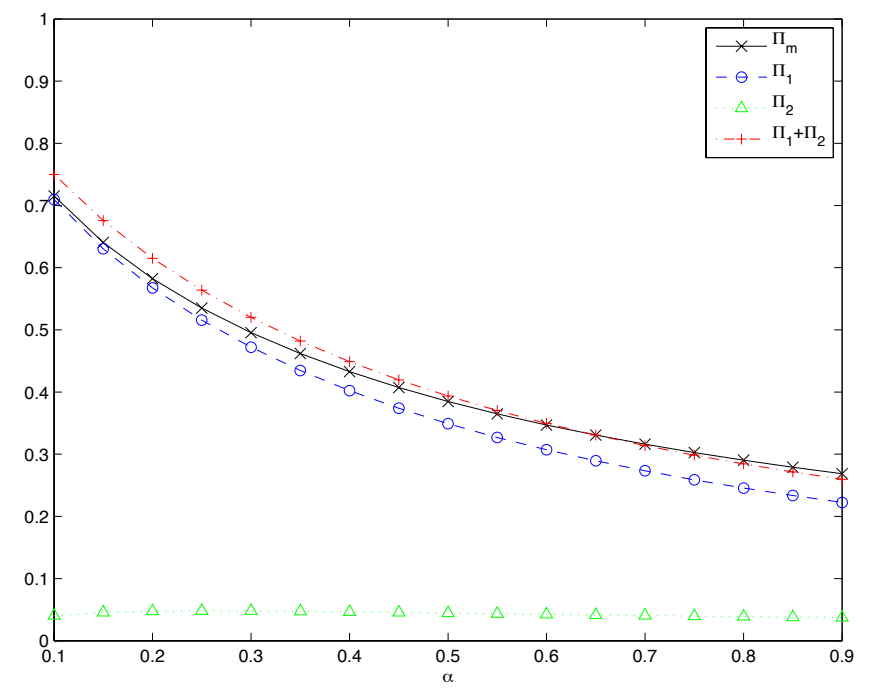

Figure 4. Equilibrium profits

\section{Entry analysis}

As stated in the discussion above, the incumbent operator is worse off when a secondary operator enters the market. We discuss in this section whether an incentive can be provided so that the former is willing to let the latter enter.

Let $m$ be a lump sum payment to be made by the entrant operator to the incumbent operator. This payment should not only make the incumbent better off, that is, $\Pi_{1}+m \geq \Pi_{m}$, but also allow the entrant to make non-negative profits, that is, $\Pi_{2}-m \geq 0$. Joining the two condition we obtain $\Pi_{m}-\Pi_{1} \leq$ $m \leq \Pi_{2}$. Thus, a necessary (and sufficient) condition for the existence of a feasible payment is

$$
\Pi_{1}+\Pi_{2} \geq \Pi_{m}
$$

In other words, if the total profit in the duopoly case is at least the same as in the monopoly case, both operators might be able to agree to redistribute their profits (via a lump sum payment) in such a way that both of them win with respect to the monopolistic situation; and will not be able to agree otherwise. As Fig. 4 shows, this condition is fulfilled for a range of values of $\alpha$ up to $\alpha \approx 0.65$.

\section{CAPACITY LEASING}

As an alternative scenario to the one modeled so far, the incumbent operator may lease an amount $\mu_{2}$ of its capacity $\mu$, to the entrant operator, keeping an amount $\mu_{1}=\mu-\mu_{2}$. Under this leasing agreement, Operator $i$ provides service using an exclusive capacity $\mu_{i}$. This scenario is shown in Fig. 5. Expression for each flow's utility and each operator's profit are the following:

$$
\begin{aligned}
u_{i} & =c T_{i}^{-\alpha}-p_{i} \\
\Pi_{i} & =\frac{\rho_{i}}{\overline{x_{i}}} p_{i}=\rho_{i} \mu_{i} p_{i} .
\end{aligned}
$$

Due to the lack of interaction between the service received by packets from each flow, first, $T_{i}$ can be computed as the 


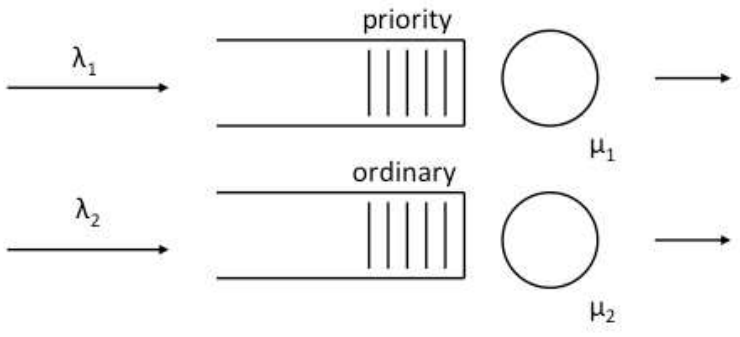

Figure 5. Queue model for the leasing agreement

mean service time of an $\mathrm{M} / \mathrm{M} / 1$ queue with $\mu_{i}$ capacity, that is, $T_{i}=\frac{1}{\mu_{i}\left(1-\rho_{i}\right)}$; and second, Operator $i$ behaves as a monopolist with respect to the flow $i$, so that (12), (13) and (14) from Section IV can be reused:

$$
\begin{aligned}
\rho_{i} & =\frac{1}{1+\alpha} \\
p_{i} & =c\left(\frac{\alpha \mu_{i}}{1+\alpha}\right)^{\alpha} \\
\Pi_{i} & =\rho_{i} \mu_{i} p_{i}=c \mu_{i}^{1+\alpha} \frac{\alpha^{\alpha}}{(1+\alpha)^{1+\alpha}} .
\end{aligned}
$$

Note the following results. First, the system is able to carry the same traffic as the monopolist. Actually, using (44), and comparing with (12)

$$
\begin{aligned}
\left(\lambda_{1}+\lambda_{2}\right) \bar{x} & =\left(\rho_{1} \mu_{1}+\rho_{2} \mu_{2}\right) \bar{x}=\frac{1}{1+\alpha}\left(\mu_{1}+\mu_{2}\right) \bar{x} \\
& =\rho_{m} \mu \bar{x}=\rho_{m} .
\end{aligned}
$$

And, from Section V-B, we know that the opportunistic access solution is able to carry more traffic than the monopolist.

Second, the system achieves lower total profits than the monopolist. Actually, using (46) and comparing with (14)

$$
\begin{aligned}
\Pi_{1}+\Pi_{2} & =c \frac{\alpha^{\alpha}}{(1+\alpha)^{1+\alpha}}\left(\mu_{1}^{1+\alpha}+\mu_{2}^{1+\alpha}\right) \\
\Pi_{m} & =c \frac{\alpha^{\alpha}}{(1+\alpha)^{1+\alpha}}\left(\mu_{1}+\mu_{2}\right)^{1+\alpha} \\
\Pi_{1}+\Pi_{2} & \leq \Pi_{m}
\end{aligned}
$$

which does not comply with (41).

The above results allows us to conclude, respectively, that the leasing solution cannot carry as much traffic as the opportunistic solution analized in Section V; and that there is no feasible payment to provide incentives to the incumbent operator. Thus, the leasing solution is inefficient and is not incentive compatible.

\section{CONCLUSIONS AND FURTHER WORK}

A scenario where a primary operator and a secondary operator provide service in a cognitive radio network has been modeled. The economic viability of supporting the secondary operator service using an opportunistic access to the spectrum owned by the primary operator has been assessed. Against the benchmark of the primary operator operating as a monopolist, we conclude that the entry of the secondary operator is desirable from an efficiency perspective, since the carried traffic increases. Additionally, for a range of parameter values, a lump sum payment can be designed so that the incumbent operator has an incentive to let the secondary operator enter. The opportunistic access setting has been compared against a leasing-based alternative, and we have concluded that the former outperforms the latter.

Future lines of work may include to model packet heterogeneity in utility and to analyze a mixed setting where part of the capacity is exclusively leased and part of it is shared under a priority scheme.

\section{ACKNOWLEDGMENT}

This work has been supported by the Spanish "Ministerio de Economía y Competitividad" through project TIN201021378-C02-02.

\section{REFERENCES}

[1] P. Ahokangas, M. Matinmikko, S. Yrjola, H. Okkonen, and T. Casey, "Simple rules for mobile network operators' strategic choices in future cognitive spectrum sharing networks," Wireless Communications, IEEE, vol. 20, no. 2, pp. 20-26, 2013.

[2] E. Commission, "Promoting the shared use of radio spectrum resources in the internal market," COM, vol. 2012, no. 478, 2012.

[3] A. Al Daoud, M. Alanyali, and D. Starobinski, "Pricing strategies for spectrum lease in secondary markets," IEEE/ACM Transactions on Networking (TON), vol. 18, no. 2, pp. 462-475, 2010.

[4] D. Niyato and E. Hossain, "Competitive spectrum sharing in cognitive radio networks: a dynamic game approach," Wireless Communications, IEEE Transactions on, vol. 7, no. 7, pp. 2651-2660, 2008.

[5] _ - "Competitive pricing for spectrum sharing in cognitive radio networks: Dynamic game, inefficiency of nash equilibrium, and collusion," Selected Areas in Communications, IEEE Journal on, vol. 26, no. 1, pp. 192-202, 2008.

[6] L. Guijarro, V. Pla, and J. R. Vidal, "Competition in cognitive radio networks: spectrum leasing and innovation," in Proceedings of CCNC. IEEE, 2011, pp. 1112-1113.

[7] L. Guijarro, V. Pla, B. Tuffin, P. Maillé, and J. R. Vidal, "Competition and bargaining in wireless networks with spectrum leasing," in Proceedings of GLOBECOM. IEEE, 2011, pp. 1-6.

[8] S. Shenker, "Fundamental design issues for the future internet," Selected Areas in Communications, IEEE Journal on, vol. 13, no. 7, pp. 11761188, 1995.

[9] H. Mendelson and S. Whang, "Optimal incentive-compatible priority pricing for the $\mathrm{m} / \mathrm{m} / 1$ queue," Operations Research, vol. 38, no. 5, pp. 870-883, 1990 .

[10] A. Odlyzko, "Paris metro pricing for the internet," in Proceedings Conference on Electronic commerce. ACM, 1999, pp. 140-147.

[11] M. Mandjes, "Pricing strategies under heterogeneous service requirements," Computer Networks, vol. 42, no. 2, pp. 231-249, 2003.

[12] Y. Hayel, D. Ros, and B. Tuffin, "Less-than-best-effort services: Pricing and scheduling," in Proceedings of INFOCOM, vol. 1. IEEE, 2004.

[13] Y. Hayel and B. Tuffin, "Pricing for heterogeneous services at a discriminatory processor sharing queue," in Proceedings of Networking Conference. IFIP, 2005.

[14] L. G. Ortuzar et al., Modelling transport. Wiley. com, 2011.

[15] M. J. Osborne, An introduction to game theory. Oxford University Press New York, 2004, vol. 3, no. 3.

[16] C.-H. Ng and S. Boon-Hee, Queueing modelling fundamentals: With applications in communication networks. Wiley, 2008. 